\title{
Comparing health-related quality of life of Dutch and Chinese patients with traumatic brain injury: do cultural differences play a role?
}

Maryse C. Cnossen ${ }^{1 *}$, Suzanne Polinder ${ }^{1}$, Pieter E. Vos ${ }^{2}$, Hester F. Lingsma ${ }^{1}$, Ewout W. Steyerberg ${ }^{1}$, Yanming Sun ${ }^{3}$, Pengpeng $\mathrm{Ye}^{4}$, Leilei Duan ${ }^{4,5}$ and Juanita A. Haagsma ${ }^{1,5}$

\begin{abstract}
Background: There is growing interest in health related quality of life (HRQoL) as an outcome measure in international trials. However, there might be differences in the conceptualization of HRQoL across different sociocultural groups. The objectives of current study were: (I) to compare HRQoL, measured with the short form (SF)-36 of Dutch and Chinese traumatic brain injury (TBI) patients 1 year after injury and; (II) to assess whether differences in SF-36 profiles could be explained by cultural differences in HRQoL conceptualization. TBI patients are of particular interest because this is an important cause of diverse impairments and disabilities in functional, physical, emotional, cognitive, and social domains that may drastically reduce HRQoL.
\end{abstract}

Methods: A prospective cohort study on adult TBI patients in the Netherlands (RUBICS) and a retrospective cohort study in China were used to compare HRQoL 1 year post-injury. Differences on subscales were assessed with the Mann-Whitney U-test. The internal consistency, interscale correlations, item-internal consistency and item-discriminate validity of Dutch and Chinese SF-36 profiles were examined. Confirmatory factor analysis was performed to assess whether Dutch and Chinese data fitted the SF-36 two factor-model (physical and mental construct).

Results: Four hundred forty seven Dutch and 173 Chinese TBI patients were included. Dutch patients obtained significantly higher scores on role limitations due to emotional problems $(p<.001)$ and general health $(p<.001)$, while Chinese patients obtained significantly higher scores on physical functioning $(p<.001)$ and bodily pain $(p=.001)$. Scores on these subscales were not explained by cultural differences in conceptualization, since item- and scale statistics were all sufficient. However, differences among Dutch and Chinese patients were found in the conceptualization of the domains vitality, mental health and social functioning.

Conclusions: One year after TBI, Dutch and Chinese patients reported a different pattern of HRQoL. Further, there might be cultural differences in the conceptualization of some of the SF-36 subscales, which has implications for outcome evaluation in multi-national trials.

Keywords: Quality of life, SF-36, Traumatic brain injury, Cultural comparison, Health domains

\footnotetext{
* Correspondence: m.c.cnossen@erasmusmc.nl

${ }^{1}$ Center for Medical Decision Sciences, Department of Public Health, Erasmus

Medical Center, P.O. Box 2040, 3000 CA Rotterdam, The Netherlands

Full list of author information is available at the end of the article
} 


\section{Background}

Health-related quality of life (HRQoL) reflects an individual's perception of how an illness and its treatment affect physical, mental and social aspects of his/her life [1]. Because it provides well-standardized information on recovery patterns, frequency, nature, and predictors of disabilities, HRQoL has been recognized as an important outcome in many medical fields, including injury [2]. Similarly, there is growing interest in international HRQoL assessment as a result of the increasing number of international trials [3].

Traumatic brain injury (TBI) is a major public health concern with a rising incidence all over the globe. In Europe, the annual number of hospital admissions is estimated at 262 per 100,000 population [4]. In other parts of the world, data on TBI incidence is less often collected systematically. Nevertheless, a 2004 epidemiological study in Eastern China found that the incidence of TBI among 77 hospitals was substantial [5]. TBI is an important cause of impairments and disability in functional, physical, emotional, cognitive, and social domains that may drastically reduce HRQoL [6, 7]. As a consequence, HRQoL has been emerged as an important outcome measurement following TBI [8].

Previous literature has indicated that there might be differences in the experience and conceptualization of HRQoL across different socio-cultural groups [9-14]. For example, in Western countries body and mind are usually regarded as two different entities, whereas Asian cultures have a more holistic sense among body and mind [15]. Therefore, the strict dichotomization of physical versus mental health, which is often included in HRQoL assessment, might not be applicable to Asian cultures [9, 12]. Also, previous evaluations of the short form (SF)-36 among Asians have shown that they conceptualize social role functioning differently from Western populations [9, 10, 12, 16, 17]. For example, Asians are more directed towards others and the use of "sickness" as an excuse for avoiding social and labour responsibilities is considered unacceptable in the Asian culture $[10,15]$. Furthermore, while Western populations associate energy level strongly with physical health, Asians associate energy more strongly with mental health $[10-13,18]$.

To our knowledge, there is no previous study that directly compared HRQoL between Western and Asian patients after injury. The purpose of this study was to compare HRQoL, measured with the SF-36, of Dutch and Chinese TBI patients 1 year after the injury. Secondly, we aimed to assess whether potential differences in SF-36 profiles between these patients could be explained by cultural differences in HRQoL conceptualization.

\section{Methods}

This study was conducted and reported according to the 'Strengthening the Reporting of Observational Based Studies' (STROBE) statement version 4 [19].

\section{Participants}

Data for the current study were obtained from two cohort studies performed in the Netherlands and China. The Radboud University Brain Injury Cohort Study (RUBICS) includes patients aged 16 years and older with mild, moderate and severe TBI presenting at the emergency department (ED) of a level I trauma center in Nijmegen, the Netherlands. Patient demographics, clinical characteristics as well as outcome measurements after 12 months follow-up were prospectively collected between June 2003 and June 2010. More information on data collection and included patients can be found in previous publications [20-24]. Data on Chinese patients were obtained from a retrospective study on injury patients admitted to one of three national injury surveillance hospitals in Zhuhai, Guangdong Province, China between January and December 2006. Patients were 15 years or older and were examined at 12 months postinjury. Data on age, gender and injury severity were collected from the hospital database. No other baseline and injury characteristics that might be relevant in the current study (e.g., education, Glasgow Coma Scale) were measured. More information about this study can be found in a previous publication [25].

To warrant comparability of patient groups, the following inclusion criteria to determine eligibility for current study were used: age $\geq 16$ years, admitted to the hospital with a clinical diagnosis of TBI, provision of informed consent and completion of at least all items of one SF-36 subscale after 12 months follow-up. Patients referred home after the ED visit and patients who died within the first year post-injury were excluded.

\section{TBI definition and classification}

In the Dutch dataset, all patients sustained a TBI. Consequently, all patients meeting the inclusion criteria for the current study were included in the analyses. The Chinese dataset was not restricted to patients with TBI, but contained patients with various injuries. The TBI patients were selected by including all patients with an International Classification of Diseases and Related Health Problems (ICD-10) code of S06, referring to traumatic intracranial injury.

Severity of TBI was determined by the Abbreviated Injury Scale - Head (AISH). The AISH is, together with the Glasgow Coma Scale (GCS), the most commonly used index of severity in TBI [26]. Severity of TBI is ranked on a scale from 1 to 6 in which 1 being mild, 2 being moderate and 6 being unsurvivable [27]. Patients 
were classified into mild/moderate and severe TBI according to their AISH score (1-2 versus $>2$ ).

The Chinese dataset did not report data on AISH. However, ICD-10 codes can be translated into AISH scores by using the ICD/AIS MAP [28, 29]. Consequently, those patients with ICD-10 codes of S06.0, S06.1, S06.2 and S06.9 were classified as having mild or moderate TBI and those with ICD-10 codes of S06.3, S06.4, S06.5, S06.6, S06.7 and S06.8 were classified as having severe TBI.

\section{Measurement of HRQoL}

The SF-36 was used to measure 12-month HRQoL. The SF-36 is the most frequently used generic instrument for HRQoL [30] and has adequate internal consistency and validity in TBI patients [31, 32]. The questionnaire has been translated and tested in more than 50 languages [30], including Dutch [33] and Cantonese [30]. The SF36 has two versions (version 1 and version 2) that differ slightly in wording, lay-out and the fact that the role questions have a dichotomous answer category in version 1 and a 5-point scale in version 2 .

The SF-36 yields a profile of the following eight concepts: physical functioning (PF), role limitations related to physical health problems (RP), bodily pain (BP), general health perceptions $(\mathrm{GH})$, vitality $(\mathrm{VT})$, social role functioning (SF), role limitations related to emotional health problems (RE) and mental health (MH). The raw scores for each concept were transformed into a 0-100 scale in which higher scores indicated better HRQoL.

In the Dutch dataset, the 12-month SF-36 version 1 was administered by a postal questionnaire that was sent to all patients. In the Chinese dataset, the 12-month SF36 version 1 was administered by a telephone interview. Patients were interviewed by a hospital nurse who received specific interview training [25].

\section{Statistical analyses}

Differences between patients included in the study and those lost to follow-up were calculated using the nonparametric Mann-Whitney $U$ test for continuous data and Chi square test for categorical data. Similarly, Dutch and Chinese patients included in this study were compared using these statistical tests on age, gender and TBI severity.

Means, standard deviations, medians, interquartile ranges and the percentage of patients with the highest ("ceiling") and lowest ("floor") scores on the SF-36 subscales were calculated for Dutch and Chinese patients classified by TBI severity. Since the number of severe TBI patients in the Chinese dataset was small $(n=20)$, the analyses were continued with mild and moderate TBI only.
Differences in SF-36 subscales between the Dutch and Chinese patients were calculated with the MannWhitney $U$ test, since all subscales had a skewed distribution. To allow for multiple testing, a stringent $p$-value of 0.0065 ( 0.05 divided by 8 subscales) was considered statistically significant. To assess whether differences between Dutch and Chinese patients could be explained by age differences between both populations, the sample was stratified into three equal age groups based on percentiles (33th and 66th) in the total population and the analyses were repeated accordingly. Since sample sizes of the age cohorts were small, statistically significance was assessed on both the stringent $p$-value $(p<.0065)$ and the standard $p$-value $(p<.05)$.

To examine whether there were differences in cultural conceptualization of HRQoL among Dutch and Chinese patients, the psychometric assumptions underlying the construction of the SF-36 were assessed for both Dutch and Chinese patients. Therefore, the reliability coefficient ("Cronbach's alpha") for each subscale was estimated. Adequate internal consistency was defined as a reliability coefficient $\geq 0.70$ [34]. Additionally, the reliability coefficient of each subscale should be larger than the subscale's interscale correlations with all other subscales [35].

Item-internal consistency and item-discriminate validity of the 35 items in both datasets were subsequently assessed. One item ("health change") was excluded since this provides an indication of perceived change in health rather than the health status 1 year post-injury. The correlation between each item and its hypothesized subscale ("corrected item-to-scale correlation") should be at least 0.40 for adequate item-internal consistency [35, 36]. Item-discriminate validity was considered adequate if the correlation between an item and its hypothesized scale was larger than the correlations between that item and all other subscales [35].

To examine whether Dutch and Chinese SF-36 subscales reflected the same underlying dimensions, i.e., a physical and mental dimension [30, 37], confirmatory factor analysis (CFA) with two latent constructs was performed. Based on theory and research in Western populations, it was hypothesized that the PF, RP and $\mathrm{BP}$ subscales were associated with the physical construct, whereas the MH, RE and SF subscales were associated with the mental construct [30,37]. For VT and GH it was expected that they load equally on both components $[30,37]$. To achieve model identification, for every latent variable one factor loading was fixed to one $\mathrm{PH}$ for physical construct; $\mathrm{MH}$ for mental construct; Additional file 1). Maximum likelihood methods were used to estimate the associations between subscales and latent factors. The Tucker-Lewis Index (TLI; recommended $>$ 0.95), Comparative Fit Index (CFI; recommended $>0.95$ ) 
and the Root mean Square Error of Approximation (RMSE; recommended $<0.08$ ) were used to examine model fit, as recommended by previous research [38]. The CFA analyses were performed using the Analysis of Moment Structures (AMOS) version 4 statistical software package. All other analyses were performed using Statistical Package for the Social Sciences (SPSS) version 21.

\section{Results}

\section{Study population}

The Dutch dataset consists of 2286 TBI patients. Of these patients, 223 were excluded because they were younger than 16 years and 804 patients were subsequently excluded because they did not receive the follow-up questionnaires because of various reasons (e.g., dementia, unknown address). Three hundred sixty patients were further excluded because they were not admitted to the hospital after the ED visit. This results in 899 eligible patients of whom 447 completed all items of at least one of the SF-36 subscales after 12month follow-up. Patients with a missing 12-month SF36 did not differ from those included in this study on age and gender. Those lost to follow-up were however less often diagnosed with severe TBI $(p<.01)$. Of the included patients, $64 \%$ was male and the median age was 46 years (interquartile range 27-58). Half of the patients had an AISH of 1-2, indicating mild and moderate TBI.

The Chinese dataset comprises information on 3664 injury patients of whom 695 patients were diagnosed with TBI according to their ICD-10 codes. Fourty-five patients were removed since they were younger than 16 years of age. Of the 650 eligible patients, 173 (27\%) completed the 12 month follow-up assessment. The main reason for non-inclusion in the study was that the telephone number was not available in the hospital database [25]. Respondents were significantly older ( median age respondents $=36$; median age nonrespondents $=32, p=.01$ ) and less often diagnosed with severe TBI (respondents: $12 \%$ severe TBI, nonrespondents: $18 \%$ severe TBI, $p=0.04$ ). Median age of the included patients $(n=173)$ was 35 years (interquartile range $24-50$ ) and $67 \%$ of the study population was male. The large majority ( $88 \%$ ) had an AISH of 1 or 2 (mild or moderate TBI).

Dutch and Chinese patients did not differ in terms of gender. Dutch patients were however significantly older than Chinese patients $(p<.001)$ and were significantly more often diagnosed with severe TBI $(p<.001)$. Comparison of other demographic and clinical characteristics between patient groups was not possible since these were not measured in the Chinese data.

\section{SF-36 scores of Dutch and Chinese patients}

Scores on SF-36 subscales for Dutch and Chinese patients, stratified by TBI severity, are presented in Table 1 . Generally, severe TBI patients seemed to report more problems with HRQoL than mild and moderate TBI patients. Ceiling effects were prominent for both Dutch and Chinese patients; more than half of the patients obtained a maximum score for role limitations due to physical problems. In the Dutch dataset, the strong ceiling effect was also shown for role limitations due to emotional problems, while in the Chinese dataset more than half of the patients obtained a maximum score for physical functioning. Since the Chinese dataset included 20 patients with severe TBI, all subsequent analyses were performed for only those patients with mild and moderate TBI.

When using the stringent $p$-value $(p<.0065)$, Chinese patients obtained significantly higher scores on the subscales PF $(p<.001)$ and BP $(p=.001)$, while Dutch patients obtained higher scores on $\mathrm{RE}(p<.001)$ and $\mathrm{GH}$ $(p<.001$; see Fig. 1 and Additional file 2$)$. Chinese patients also obtained higher scores on SF $(p=.026)$, but this was not statistically significant using the stringent $p$ value.

Age differences between Dutch and Chinese patients did not explain the differences in the PF and RE scale scores, since differences remained statistically significant in the different age cohorts $(p<.0065$ in two age cohorts; $p<.05$ in one age cohort, see Additional file 2). For BP, however, the statistically significant differences between Dutch and Chinese patients did not withstand after stratification for age (no significant differences between Dutch and Chinese patients in 2 out of 3 age strata, see Additional file 2). With regard to GH, Dutch patients obtained significantly higher scores in two out of three age cohorts $(p<.0065)$. In the youngest age cohort, however, no statistically significant differences were found between Dutch and Chinese patients.

\section{Cultural conceptualization of $\mathrm{HRQOL}$}

In the Dutch dataset, all SF-36 subscales had an adequate internal consistency and none of the intercorrelations between subscales were larger than the values of Cronbach's alpha (see Table 2). Item-internal consistency and item-discriminate validity were also adequate for all items. One of the items of the vitality scale (VT1), nevertheless, correlated higher with the $\mathrm{MH}$ scale $(\mathrm{r}=$ $0.55)$ than with the VT scale itself $(r=0.49$; see Additional file 3).

In the Chinese dataset, internal consistency was insufficient for two subscales (VT and SF). Also, the intercorrelations between SF and six other subscales were larger than the value of Cronbach's alpha for the SF scale. Item-internal consistency and item-discriminate validity 
Table 1 Short Form (SF)-36 scores of Dutch and Chinese traumatic brain injury patients 12 months post-injury

Nijmegen, the Netherlands

Abbreviated Injury Score Head 1-2

\begin{tabular}{llllll} 
& N & Range & Mean (SD) & Median (IQR) & Floor (\%) \\
PF & 200 & $0-100$ & $81.2(24.6)$ & $95(70-100)$ & $0.5 \%$ \\
RP & 211 & $0-100$ & $68.1(40.7)$ & $100(25-100)$ & $19.9 \%$ \\
BP & 217 & $0-100$ & $73.9(26.2)$ & $80(52-100)$ & $0.9 \%$ \\
GH & 213 & $0-100$ & $68.5(22.8)$ & $72(52-87)$ & $0.5 \%$ \\
VT & 215 & $5-100$ & $65.0(21.3)$ & $65(50-80)$ & $0 \%$ \\
SF & 217 & $13-100$ & $81.5(22.2)$ & $88(63-100)$ & $0 \%$ \\
RE & 214 & $0-100$ & $81.5(34.2)$ & $100(67-100)$ & $11.7 \%$ \\
MH & 216 & $0-100$ & $74.5(20.2)$ & $80(64-88)$ & $0.5 \%$ \\
\multicolumn{7}{l}{ Zhuhai, China }
\end{tabular}

Abbreviated Injury Score Head 1-2

$\begin{array}{lllllll} & \text { N } & \text { Range } & \text { Mean (SD) } & \text { Median (IQR) } & \text { Floor (\%) } & \text { Ceiling (\%) } \\ \text { PF } & 153 & 5-100 & 93.0(16.8) & 100(95-100) & 0 \% & 64.7 \% \\ \text { RP } & 153 & 0-100 & 68.8(40.6) & 100(25-100) & 19.6 \% & 56.2 \% \\ \text { BP } & 153 & 0-100 & 81.7(26.4) & 100(67-100) & 1.3 \% & 56.9 \% \\ \text { GH } & 153 & 5-100 & 58.0(23.9) & 60(40-75) & 0 \% & 2.6 \% \\ \text { VT } & 153 & 0-100 & 66.6(23.8) & 70(50-85) & 1.3 \% & 7.8 \% \\ \text { SF } & 153 & 11-100 & 85.0(21.7) & 89(78-100) & 0 \% & 49.7 \% \\ \text { RE } & 153 & 0-100 & 55.3(41.0) & 67(0-100) & 26.8 \% & 37.3 \% \\ \text { MH } & 153 & 4-100 & 75.6(20.4) & 80(64-90) & 0 \% & 11.8 \%\end{array}$

Note: Scale scores range from 0 to 100 , with 100 representing optimal functioning

Abbreviations: SD Standard deviation, IQR Interquartile range, PF Physical functioning, RP Role physical, $B P$ Bodily pain, GH General health, VT Vitality, SF Social functioning, RE Role-emotional, $M H$ Mental health

${ }^{a}$ Floor (\%) refers to the percentage of patients with the lowest score on a subscale (score 0); Ceiling (\%) refers to the percentage of patients with the highest score on a subscale (score 100)

were adequate for the large majority of items. However, four items (VT2, SF1, SF2 and MH3) obtained a corrected item-to-scale correlation below 0.40. Furthermore, some items from the GH, VT, SF and $\mathrm{MH}$ subscales correlated higher with other subscales than with their own hypothesized subscales (see Additional file 3).

CFA with a two-factor model in the Dutch population resulted in a TLI of 0.88 , a CFI of 0.95 and an RMSEA of 0.13 , indicating a mixed pattern of model fit. The associations between the SF-36 subscales and the two latent constructs was as hypothesized for seven subscales (Table 3). The VT subscale, however, was strongly associated with the mental component $(\beta=1.08, p<.01)$ but not with the physical component $(B=0.01, p=0.94)$. The association between the physical and mental health construct was strong in the Dutch data $(r=0.70)$.

CFA with a two-factor model in the Chinese population had an adequate model fit (TLI: 0.95, CFI: 0.97 and RMSE: 0.08$)$. However, the VT scale was negatively associated with the physical construct $(B=-2.31, p=.18)$ and the association between the mental construct and
Abbreviated Injury Score Head $>2$

$\begin{array}{llllll}\text { N } & \text { Range } & \text { Mean (SD) } & \text { Median (IQR) } & \text { Floor (\%) } & \text { Ceiling (\%) } \\ 207 & 0-100 & 77.5(28.5) & 90(70-100) & 4.8 \% & 30.0 \% \\ 214 & 0-100 & 56.7(43.9) & 75(0-100) & 29.9 \% & 44.4 \% \\ 216 & 0-100 & 75.9(25.2) & 82(62-100) & 1.4 \% & 40.7 \% \\ 216 & 10-100 & 70.2(20.0) & 72(60-87) & 0 \% & 4.2 \% \\ 218 & 5-100 & 64.6(20.3) & 65(50-80) & 0 \% & 4.6 \% \\ 220 & 13-100 & 78.3(22.5) & 88(63-100) & 0 \% & 37.3 \% \\ 217 & 0-100 & 75.6(38.1) & 100(67-100) & 16.1 \% & 65.9 \% \\ 218 & 20-100 & 73.8(20.1) & 80(63-88) & 0 \% & 6.0 \%\end{array}$

Abbreviated Injury Score Head $>2$

$\begin{array}{llllll}\text { N } & \text { Range } & \text { Mean (SD) } & \text { Median (IQR) } & \text { Floor (\%) } & \text { Ceiling (\%) } \\ 20 & 0-100 & 82.3(26.2) & 95(75-100) & 5.0 \% & 45 \% \\ 20 & 0-100 & 60.0(44.7) & 88(6-100) & 25.0 \% & 50 \% \\ 20 & 10-100 & 70.8(27.8) & 79(52-97) & 0 \% & 25 \% \\ 20 & 15-85 & 51.5(19.5) & 53(40-65) & 0 \% & 0 \% \\ 20 & 15-100 & 67.5(24.8) & 78(46-85) & 0 \% & 10 \% \\ 20 & 33-100 & 80.6(23.9) & 89(58-100) & 0 \% & 45 \% \\ 20 & 0-100 & 60.0(44.1) & 67(0-100) & 30.0 \% & 45 \% \\ 20 & 48-100 & 81.0(14.0) & 82(72-92) & 0 \% & 10 \%\end{array}$




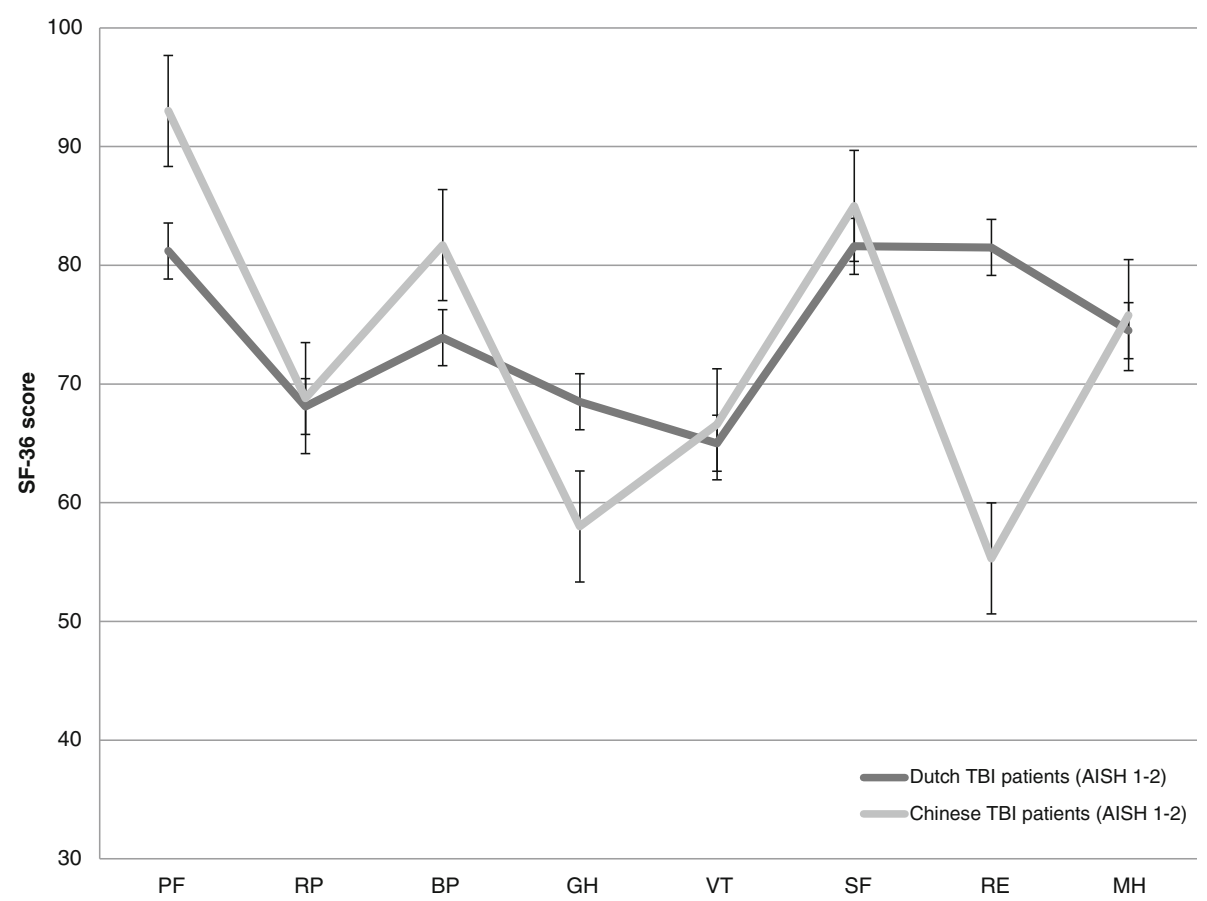

Fig. 1 Short Form (SF)-36 score profiles of Dutch and Chinese patients. Note. Figure shows SF-36 score profiles of Dutch and Chinese patients with mild and moderate traumatic brain injury 12 months post-injury. Scale scores range from 0 to 100, with 100 representing optimal functioning. Abbreviations. $\mathrm{PF}=$ physical functioning; $\mathrm{RP}=$ role physical; $\mathrm{BP}=$ bodily pain; $\mathrm{GH}=$ general health; $\mathrm{VT}=$ vitality; $\mathrm{SF}=$ social functioning; $\mathrm{RE}=$ roleemotional; $\mathrm{MH}=$ mental health; AISH = Abbreviated Injury Scale Head

these differences. Firstly, Dutch and Chinese patients might value similar symptoms and limitations differently. In China, health is usually described as a balance between "yin and yang" and the appreciation of one's health is largely influenced by spirituality [15]. In the Dutch culture, on the opposite, HRQoL might be more related to the number and severity of symptoms. In addition, because cultural values emphasize harmony in Asian cultures, Asians might be more optimistic when experiencing similar symptoms and less likely to report negative and extreme feelings [41]. Related, coping strategies of Dutch and Chinese patients might vary, since these are largely influenced by cultural systems [9]. Another hypothesis might be that the differences between Dutch and Chinese patients, especially in the physical health dimension, reflect the variation in acute and rehabilitation treatment between countries. In China, a part of the TBI related care is not reimbursed [42] and therefore, it is possible that some of the Chinese patients included in this study did not receive adequate acute or

Table 2 Reliability coefficients (in diagonals) and Pearson's correlation coefficients between Short Form (SF)-36 subscales in patients with mild and moderate traumatic brain injury

\begin{tabular}{|c|c|c|c|c|c|c|c|c|c|c|c|c|c|c|c|c|c|}
\hline \multicolumn{9}{|c|}{ Nijmegen, the Netherlands } & \multicolumn{9}{|c|}{ Zhuhai, China } \\
\hline & $\mathrm{PF}$ & $\mathrm{RP}$ & $\mathrm{BP}$ & $\mathrm{GH}$ & VT & SF & RE & $\mathrm{MH}$ & & PF & $\mathrm{RP}$ & $\mathrm{BP}$ & $\mathrm{GH}$ & $\mathrm{VT}$ & SF & RE & $\mathrm{MH}$ \\
\hline$\overline{P F}$ & $(.94)$ & & & & & & & & PF & $(.93)$ & & & & & & & \\
\hline $\mathrm{RP}$ & $.73^{* *}$ & $(.90)$ & & & & & & & $\mathrm{RP}$ & $.53^{* *}$ & $(.90)$ & & & & & & \\
\hline $\mathrm{BP}$ & $.64^{* *}$ & $.71^{* *}$ & $(.88)$ & & & & & & $\mathrm{BP}$ & $.47^{* *}$ & $.68^{* *}$ & $(.90)$ & & & & & \\
\hline $\mathrm{GH}$ & $.54^{* *}$ & $.68^{* *}$ & $.56^{* *}$ & $(.83)$ & & & & & $\mathrm{GH}$ & $.44^{* *}$ & $.69^{* *}$ & $.61^{* *}$ & $(.76)$ & & & & \\
\hline VT & $.41^{* *}$ & $.60^{* *}$ & $.50^{* *}$ & $.66^{* *}$ & $(.75)$ & & & & VT & $.30^{* *}$ & $.50^{* *}$ & $.43^{* *}$ & $.51^{* *}$ & $(.66)$ & & & \\
\hline SF & $.54^{* *}$ & $.67^{* *}$ & $.54^{* *}$ & $.63^{* *}$ & $.73^{* *}$ & (.83) & & & SF & $.53^{* *}$ & $.64^{* *}$ & $.61^{* *}$ & $.59^{* *}$ & $.50^{* *}$ & $(.49)$ & & \\
\hline RE & $.38^{* *}$ & $.59^{* *}$ & $.37^{* *}$ & $.47^{* *}$ & $.55^{* *}$ & $.66^{* *}$ & $(.86)$ & & RE & $.26^{* *}$ & $.56^{* *}$ & $.41^{* *}$ & $.49^{* *}$ & $.47^{* *}$ & $.45^{* *}$ & (.78) & \\
\hline $\mathrm{MH}$ & $.31^{* *}$ & $.50^{* *}$ & $.35^{* *}$ & $.61^{* *}$ & $.78^{* *}$ & $.72^{* *}$ & $.61^{* *}$ & (.89) & $\mathrm{MH}$ & $.28^{* *}$ & $.47^{* *}$ & $.40^{* *}$ & $.47^{* *}$ & $.63^{* *}$ & $.51^{* *}$ & $.47^{* *}$ & (.70) \\
\hline
\end{tabular}

Note. Table shows reliability coefficients and Pearson's correlation coefficients between SF-36 subscales in patients with mild and moderate traumatic brain injury 12 months post-injury

Abbreviations: PF Physical functioning, RP Role physical, BP Bodily pain, GH General health, VT Vitality, SF Social functioning, RE Role-emotional, MH Mental health 
Table 3 Confirmatory Factor Analysis of the Short Form (SF)-36 subscales

\begin{tabular}{|c|c|c|c|c|c|c|c|}
\hline \multirow[b]{2}{*}{ Observed variable } & \multirow[b]{2}{*}{ Latent construct } & \multicolumn{3}{|c|}{ Nijmegen, the Netherlands } & \multicolumn{3}{|c|}{ Zhuhai, China } \\
\hline & & $B$ & $B$ & $p$-value & $B$ & B & $p$-value \\
\hline$\overline{\mathrm{PF}}$ & Physical & $1.00^{\mathrm{a}}$ & 0.78 & NA & $1.00^{\mathrm{a}}$ & 0.60 & NA \\
\hline $\mathrm{RP}$ & Physical & 2.02 & 0.94 & $<.01$ & 3.58 & 0.88 & $<.01$ \\
\hline $\mathrm{BP}$ & Physical & 1.04 & 0.76 & $<.01$ & 2.04 & 0.77 & $<.01$ \\
\hline $\mathrm{GH}$ & Physical & 0.48 & 0.45 & $<.01$ & 1.25 & 0.52 & .01 \\
\hline VT & Physical & 0.01 & 0.01 & .94 & -2.31 & -0.97 & .18 \\
\hline $\mathrm{GH}$ & Mental & 0.59 & 0.45 & $<.01$ & 0.49 & 0.28 & .14 \\
\hline VT & Mental & 1.06 & 0.86 & $<.01$ & 2.87 & 1.65 & .03 \\
\hline SF & Mental & 1.13 & 0.88 & $<.01$ & 1.19 & 0.75 & $<.01$ \\
\hline RE & Mental & 1.39 & 0.70 & $<.01$ & 1.89 & 0.63 & $<.01$ \\
\hline $\mathrm{MH}$ & Mental & $1.00^{\mathrm{a}}$ & 0.86 & NA & $1.00^{\mathrm{a}}$ & 0.67 & NA \\
\hline
\end{tabular}

Note: Table represents unstandardized ( $($ ) and standardized (B) regression weights between subscales and the physical and mental component for both the Dutch and the Chinese mild and moderate traumatic brain injury patients 12 months post-injury

Abbreviations: PF Physical functioning, RP Role physical, BP Bodily pain, GH General health, VT Vitality, SF Social functioning, RE Role-emotional, MH Mental health

${ }^{\mathrm{a}}$ Regression weight was set to 1.00

*Statistically significant $(p<.05)$ association

- = strong association between subscale and rotated principal component is expected based on previous research [30, 37]

0 weak/no association between subscale and rotated principal component is expected based on previous research [30,37]

rehabilitative care, influencing their HRQoL 1 year postinjury. Lastly, the differences between Dutch and Chinese patients might also be explained by a lack of comparability of the included patients (e.g., there might have been baseline differences between patients) and study designs (prospective study with postal questionnaire versus retrospective study with telephone interview).

Our finding that social functioning is conceptualized differently among Dutch and Chinese mild and moderate TBI patients is consistent with previous research about psychometrics of the SF-36 in Asian cultures [9, $10,14,16-18]$. It has been suggested that the concept of social functioning is more Westernized and less clear for Asian people [14]. The strong association between vitality and mental health in our Chinese sample was also consistent with previous literature of the general population $[11,13,14,18]$. In traditional Chinese medicine a mental disorder is referred to as "the loss of a vital substance of spirit" [17], which could explain this strong association. Notwithstanding, we also found that vitality was strongly associated with mental health but not with physical health in the Dutch population, suggesting that this association could also be related to the TBI rather than to cultural conceptualization. The sequelae of mild and moderate TBI often includes mental health problems as well as fatigue or lack of energy [43,44], whereas physical problems, such as headache, usually resolve within a few months [45]. Since this is the first study that performed CFA with the SF-36 in a TBI population, current findings should be confirmed by future studies with larger numbers of patients. The high correlation between mental and physical health in Chinese patients may indicate that these patients have a more holistic sense among body and mind [15]. As a consequence, one latent factor rather than two (physical and mental health) might have been more appropriate for the Chinese patients. This should also be confirmed in studies with larger sample sizes.

This is the first study that directly compared HRQoL between Asian and Western patients after injury. A strength of current study is that we did not only assess differences on the SF-36 subscales between Dutch and Chinese patients, but also examined whether these differences could be explained by cultural differences in the conceptualization of quality of life. In addition, we stratified our analyses for age and severity and included an adequate sample size.

Results should however be interpreted in the light of the following limitations. First, response rates were relatively low (50\% for the Netherlands and $27 \%$ for China) for both datasets. Although low response rates do not necessarily result in bias [46], we cannot exclude that the patients in our study comprise an a-select sample. A second limitation concerns the comparability of Dutch and Chinese patients. Although patients were similar in terms of gender, and were stratified based on TBI severity and age, we cannot exclude that the patient groups differed on demographic and clinical variables (e.g., education, Glasgow Coma Scale) that were not measured in the Chinese dataset. Related, comorbidity was not assessed in both cohorts, while it is common in TBI patients $[47,48]$ and could also influence HRQoL [49]. Moreover, the Dutch study administered the SF-36 by a postal questionnaire while the Chinese study used telephone interviews, which might not be comparable. For example, in a telephone interview, social desirability bias 
is relatively likely to occur $[50,51]$, which might have resulted in more optimistic results among Chinese patients. Also, a postal questionnaire, especially in patients with severe TBI, might not be reliable because of memory and concentration problems experienced by these patients [52]. Comparability of Dutch and Chinese patients is further hampered by differences in study design; the Dutch database was a prospective cohort study whereas the Chinese dataset was retrospectively collected.

The time between injury and follow-up can also be considered a limitation in this study. Although it is known that a subset of mild and moderate TBI patients experience long-lasting symptoms $[44,49,53]$, the majority is expected to be recovered 1 year post-injury [54]. This might have caused the strong ceiling effects in our study. Ceiling effects are considered to be present if the highest score on a subscale is obtained in more than $15 \%$ of the respondents $[55,56]$, which was the case in the majority of subscales for Dutch and Chinese mild and moderate patients. Ceiling effects may reduce reliability and validity of subscales [56] and might indicate that the SF-36 lacks sensitivity to examine differences in TBI patient groups 1 year after the injury. In addition, the skewed distribution might have influenced the validity of the CFA analyses because normality is one of the assumptions of the maximum likelihood method. However, in small sample sizes $(\mathrm{N}<200)$ the maximum likelihood method outperformed other analytic methods such as diagonally weighted least squares [57].

Given these limitations, the findings of current study should be interpreted as preliminary and hypothesis generating. We therefore recommend future studies to use highly comparable patient groups in terms of demographics and clinical variables and a detailed registration of the acute and rehabilitative care provided. Additionally, the inclusion of more objective outcome measurements (e.g., Glasgow Outcome Scale Extended) might provide insight on whether Western and Asian patients experience other symptoms or interpret/cope differently with similar symptoms following injury. Related, next to the SF-36, which is a measurement of general HRQoL, a disease-specific measurement such as the QOLIBRI [58] is recommended to measure the full impact of TBI on HRQoL [59]. In addition, qualitative studies, such as interviews or focus groups might also be suitable to study cultural differences in HRQoL after injury.

Our finding that Chinese mild and moderate TBI patients conceptualize some of the subscales differently, poses a challenge for multi-national trials with HRQoL as outcome measurement. A prerequisite in multinational trials measuring health status is that the same underlying dimensions are measured and that these dimensions are culturally meaningful in all participating countries [13]. Our research shows that this can be doubted in a TBI population, which was in line with findings in the general population $[9,11]$. We therefore recommend multi-national trials including both Asian and Western countries to be cautious in their interpretation of health outcome.

\section{Conclusions}

One year after TBI, Dutch and Chinese patients reported a different pattern of HRQoL. Further, we found cultural differences in the conceptualization of some of the SF36 subscales, which has implication for outcome evaluation in multi-national trials.

\section{Additional files}

Additional file 1: Hypothesized confirmatory factor analysis. (PDF 9 kb)

Additional file 2: Comparison of Short Form (SF)-36 scales among Dutch and Chinese mild and moderate traumatic brain injury patients for the total population and stratified across three age groups. Description of data: a table that shows the $p$-values of the comparison between Dutch and Chinese mild and moderate traumatic brain injury. We first show a $p$-value in the total population and after that, we divide the population into three age groups and show the $p$-values accordingly. (DOCX 21 kb)

Additional file 3: Item Characteristics of the Short Form (SF)-36 among Dutch and Chinese mild and moderate traumatic brain injury patients. Description of data: The file contains of two tables (one for the Duch patients and one for the Chinese patients) with item characteristics. We show the mean and standard deviation of all SF-36 items, the corrected item-to-scale correlation and the correlation between the item and all other SF-36 subscales. (DOCX $27 \mathrm{~kb}$ )

\section{Abbreviations}

AISH: Abbreviated Injury Scale - Head; BP: Bodily pain; ED: Emergency department; GCS: Glasgow Coma Scale; GH: General health; HRQoL: Health related quality of life; ICD: International Classification of Diseases and Related Health Problems; MH: Mental health; PF: Physical functioning; RE: Role limitations related to emotional health problems; RP: Role limitations related to physical health problems; RUBICS: Radboud University Brain Injury Cohort Study; SF: Social functioning; SF-36: Short form 36; SPSS: Statistical package for the social sciences; STROBE: Strengthening the Reporting of

Observational Based Studies; TBI: Traumatic brain injury; VT: Vitality

\section{Acknowledgement}

Authors would like to thank Amber Hoek, MD for her help with checking the ICD/AIS conversion.

\section{Funding}

The authors Maryse Cnossen, Suzanne Polinder, Hester Lingsma, Ewout Steyerberg and Juanita Haagsma were supported by the European Union FP 7th Framework program (grant 602150).

\section{Availability of data and material}

The data that support the findings of this study are available from RUBICS and Zhuhai but restrictions apply to the availability of these data, which were used under license for the current study, and so are not publicly available. Data are however available from the authors upon reasonable request and with permission of Pieter Vos (RUBICS) and Pengpeng Ye (Chinese database).

\section{Authors' contribution}

$\mathrm{JH}$ and SP developed the study design and interpreted the data. MC analysed and interpreted the data and wrote the manuscript. PV coordinated the data collection for the RUBICS study and YS, PY and LD coordinated the 
data collection for the Chinese database. All authors critically revised the paper. All authors read and approved the final manuscript.

\section{Competing interests}

The authors declare that they have no competing interests.

\section{Consent for publication}

Not applicable.

\section{Ethics approval and consent to participate}

The RUBICS study was approved by the ethical standards committee of the Radboud University Medical Center. Written informed consent was obtained from all patients in this study.

The Chinese study was approved by the Health Bureau in Zhuhai city. Oral informed consent was obtained from all patients during the telephone interview.

\section{Publisher's Note}

Springer Nature remains neutral with regard to jurisdictional claims in published maps and institutional affiliations.

\section{Author details}

${ }^{1}$ Center for Medical Decision Sciences, Department of Public Health, Erasmus Medical Center, P.O. Box 2040, 3000 CA Rotterdam, The Netherlands. ${ }^{2}$ Department of Neurology, Slingeland Hospital, Doetinchem, The Netherlands. ${ }^{3}$ Beijing Centers for Disease Control and Prevention, Beijng, People's Republic of China. ${ }^{4}$ NCDC, China CDC, Beijing, People's Republic of China. ${ }^{5}$ nstitute for Health Metrics and Evaluation, University of Washington, Seattle, USA

Received: 29 July 2016 Accepted: 27 March 2017

Published online: 14 April 2017

\section{References}

1. von Steinbuechel N, Richter S, Morawetz C, Riemsma R. Assessment of subjective health and health-related quality of life in persons with acquired or degenerative brain injury. Curr Opin Neurol. 2005;18:681-91.

2. Neugebauer E, Bouillon B, Bullinger M, Wood-Dauphinee S. Quality of life after multiple trauma-summary and recommendations of the consensus conference. Restor Neurol Neurosci. 2002;20:161-7.

3. Al Sayah F, Ishaque S, Lau D, Johnson JA. Health related quality of life measures in Arabic speaking populations: a systematic review on crosscultural adaptation and measurement properties. Qual Life Res. 2013;22: 213-29

4. Peeters W, van den Brande R, Polinder S, Brazinova A, Steyerberg EW, Lingsma HF, Maas Al. Epidemiology of traumatic brain injury in Europe. Acta Neurochir (Wien). 2015:157:1683-96.

5. Wu X, Hu J, Zhuo L, Fu C, Hui G, Wang Y, Yang W, Teng L, Lu S, Xu G. Epidemiology of traumatic brain injury in eastern China, 2004: a prospective large case study. J Trauma. 2008;64:1313-9.

6. Andelic N, Hammergren N, Bautz-Holter E, Sveen U, Brunborg C, Roe C. Functional outcome and health-related quality of life 10 years after moderate-to-severe traumatic brain injury. Acta Neurol Scand. 2009;120:16-23.

7. Dijkers MP. Quality of life after traumatic brain injury: a review of research approaches and findings. Arch Phys Med Rehabil. 2004;85:S21-35.

8. NINDS Common Data Elements - Traumatic Brain Injury [http://www. commondataelements.ninds.nih.gov/tbi.aspx\#tab=Data_Standards]

9. Ngo-Metzger Q, Sorkin DH, Mangione CM, Gandek B, Hays RD. Evaluating the SF-36 Health Survey (Version 2) in Older Vietnamese Americans. J Aging Health. 2008:20:420-36.

10. Li L, Wang HM, Shen Y. Chinese SF-36 Health Survey: translation, cultural adaptation, validation, and normalisation. J Epidemiol Community Health. 2003:57:259-63.

11. Hinton DE, Sinclar SJ, Chung RCY, Pollack MH. The SF-36 among Cambodian and Vietnamese Refugees: An examination of psychometric properties. J Psychopathol Behav Assess. 2006;29:38-45

12. Thumboo J, Wu Y, Tai ES, Gandek B, Lee J, Ma S, Heng D, Wee HL. Reliability and validity of the English (Singapore) and Chinese (Singapore) versions of the Short-Form 36 version 2 in a multi-ethnic urban Asian population in Singapore. Qual Life Res. 2013;22:2501-8.
13. Fukuhara S, Ware Jr JE, Kosinski M, Wada S, Gandek B. Psychometric and clinical tests of validity of the Japanese SF-36 Health Survey. J Clin Epidemiol. 1998;51:1045-53.

14. Tseng HM, Lu JFR, Gandek B. Cultural issues in using the SF-36 health survey in Asia: Results from Taiwan. Health Qual Life Outcomes. 2003;1:72.

15. Torsch VL, Ma GX. Cross-cultural comparison of health perceptions, concerns, and coping strategies among Asian and Pacific Islander American elders. Qual Health Res. 2000;10:471-89.

16. Azen SP, Palmer JM, Carlson M, Mandel D, Cherry BJ, Fanchiang SP, Jackson J, Clark F. Psychometric properties of a Chinese translation of the SF-36 health survey questionnaire in the well elderly study. J Aging Health. 1999; 11:240-51.

17. Ren XS, Amick IB, Zhou L, Gandek B. Translation and psychometric evaluation of a Chinese version of the SF- 36 Health Survey in the United States. J Clin Epidemiol. 1998:51:1129-38.

18. Fuh JL, Wang SJ, Lu SR, Juang KD, Lee SJ. Psychometric evaluation of a Chinese (Taiwanese) version of the SF-36 health survey amongst middleaged women from a rural community. Qual Life Res. 2000;9:675-83.

19. STROBE statement - checklist of items that should be included in reports of observational studies [http://www.strobe-statement.org/fileadmin/Strobe/ uploads/checklists/STROBE_checklist_v4_combined.pdf]

20. Haagsma JA, Scholten AC, Andriessen TM, Vos PE, Van Beeck EF, Polinder S. Impact of depression and post-traumatic stress disorder on functional outcome and health-related quality of life of patients with mild traumatic brain injury. J Neurotrauma. 2015;32:853-62.

21. Scholten AC, Haagsma JA, Andriessen TM, Vos PE, Steyerberg EW, van Beeck EF, Polinder S. Health-related quality of life after mild, moderate and severe traumatic brain injury: patterns and predictors of suboptimal functioning during the first year after injury. Injury. 2015;46:616-24.

22. Stulemeijer M, van der Werf S, Borm GF, Vos PE. Early prediction of favourable recovery 6 months after mild traumatic brain injury. J Neurol Neurosurg Psychiatry. 2008;79:936-42

23. Jacobs B, Beems T, Van Der Vliet TM, Van Vugt AB, Hoedemaekers C, Horn J, Franschman G, Haitsma I, Van Der Naalt J, Andriessen TMJC, et al. Outcome prediction in moderate and severe traumatic brain injury: A focus on computed tomography variables. Neurocrit Care. 2013:19:79-89.

24. Vos PE, Jacobs B, Andriessen TMJC, Lamers KJB, Borm GF, Beems T, Edwards M, Rosmalen CF, Vissers JLM. GFAP and S100B are biomarkers of traumatic brain injury: An observational cohort study. Neurology. 2010;75:1786-93.

25. Sun $Y, Y e P$, Haagsma JA, Vos T, Wu F, Duan L. Functional outcome one year after injury in Zhuhai, Guangdong Province in China. 2016.

26. Corrigan JD, Selassie AW, Orman JA. The epidemiology of traumatic brain injury. J Head Trauma Rehabil. 2010;25:72-80.

27. Association for the Advancement of Automotive Medicine. The abbreviated injury scale (AIS) 1990 revision - Update 98. Association for the Advancement of Automotive Medicine: Des Plaines, II. 1998.

28. Durbin DR, Localio AR, MacKenzie EJ. Validation of the ICD/AIS MAP for pediatric use. Inj Prev. 2001;7:96-9.

29. MacKenzie EJ, Steinwachs DM, Shankar B. Classifying trauma severity based on hospital discharge diagnoses. Validation of an ICD-9CM to AIS-85 conversion table. Med Care. 1989:27:412-22

30. Ware Jr JE, Gandek B. Overview of the SF-36 Health Survey and the International Quality of Life Assessment (IQOLA) Project. J Clin Epidemiol. 1998:51:903-12

31. Guilfoyle MR, Seeley HM, Corteen E, Harkin C, Richards H, Menon DK, Hutchinson PJ. Assessing quality of life after traumatic brain injury: examination of the short form 36 health survey. J Neurotrauma. 2010;27: 2173-81.

32. Diaz AP, Schwarzbold ML, Thais ME, Hohl A, Bertotti MM, Schmoeller R, Nunes JC, Prediger R, Linhares MN, Guarnieri R, Walz R. Psychiatric disorders and health-related quality of life after severe traumatic brain injury: a prospective study. J Neurotrauma. 2012:29:1029-37.

33. Aaronson NK, Muller M, Cohen PD, Essink-Bot ML, Fekkes M, Sanderman R, Sprangers MA, te Velde A, Verrips E. Translation, validation, and norming of the Dutch language version of the SF-36 Health Survey in community and chronic disease populations. J Clin Epidemiol. 1998:51:1055-68.

34. Nunnally JC. Psychometric Theory. 2nd ed. New York: McGraw-Hill; 1978

35. Campbell DT, Fiske DW. Convergent and discriminant validation by the multitrait-multimethod matrix. Psychol Bull. 1959;56:81-105.

36. Howard Kl, Forehand GA. A method for correcting item-total correlations for the effect of relevant item inclusion. Educ Psychol Meas. 1962;22:731-5. 
37. Ware JE, Kosinski M, Keller SD. SF-36 physical and mental health summary scales: A User's manual. Boston: The Health Institute; 1994.

38. Schreiber J, Stage FK, King J, Nora A, Barlow EA. Reporting structural equation modeling and confirmatory factor analysis results: A review. J Educ Res. 2006:99:323-37.

39. Wang W, Lau Y, Chow A, Thompson DR, He HG. Health-related quality of life and social support among Chinese patients with coronary heart disease in mainland China. Eur J Cardiovasc Nurs. 2014;13:48-54.

40. Tang C, Shanmugasegaram S, Chow CM, Cho N, Tan Y, Grace SL. A comparison of psychosocial health in North American and Chinese Canadian cardiac outpatients, and ethnocultural correlates of quality of life. Ethn Dis. 2014;24:302-9.

41. Scott NW, Fayers PM, Aaronson NK, Bottomley A, de Graeff A, Groenvold M, Koller M, Petersen MA, Sprangers MA. Eortc, the Quality of Life CrossCultural Meta-Analysis G: The use of differential item functioning analyses to identify cultural differences in responses to the EORTC QLQ-C30. Qual Life Res. 2007;16:115-29.

42. Meng Q, Fang H, Liu X, Yuan B, Xu J. Consolidating the social health insurance schemes in China: towards an equitable and efficient health system. Lancet. 2015;386:1484-92.

43. Scholten AC, Haagsma JA, Cnossen MC, Olff M, Van Beeck EF, Polinder S. Prevalence and risk factors of anxiety and depressive disorders following traumatic brain injury: a systematic review. J Neurotrauma. 2016;33(22): 1969-94.

44. Sivak S, Nosal V, Bittsansky M, Dluha J, Dobrota D, Kurca E. Type and occurrence of serious complications in patients after mild traumatic brain injury. Bratisl Lek Listy. 2016;117:22-5.

45. Carroll LJ, Cassidy JD, Peloso PM, Borg J, von Holst H, Holm L, Paniak C, Pepin M. Prognosis for mild traumatic brain injury: results of the WHO Collaborating Centre Task Force on Mild Traumatic Brain Injury. J Rehabil Med. 2004;84.

46. Kristman V, Manno M, Côté P. Loss to follow-up in cohort studies: How much is too much? Eur J Epidemiol. 2004;19:751-60.

47. Sabaz M, Simpson GK, Walker AJ, Rogers JM, Gillis I, Strettles B. Prevalence, comorbidities, and correlates of challenging behavior among communitydwelling adults with severe traumatic brain injury: a multicenter study. J Head Trauma Rehabil. 2014;29:E19-30.

48. Thompson HJ, Dikmen S, Temkin N. Prevalence of comorbidity and its association with traumatic brain injury and outcomes in older adults. Res Gerontol Nurs. 2012;5:17-24.

49. Theadom A, Parag V, Dowell T, McPherson K, Starkey N, Barker-Collo S, Jones K, Ameratunga S, Feigin VL, Group BR. Persistent problems 1 year after mild traumatic brain injury: a longitudinal population study in New Zealand. Br J Gen Pract. 2016;66:e16-23.

50. Sibbald B, Addington-Hall J, Brenneman D, Freeling P. Telephone versus postal surveys of general practitioners: methodological considerations. $\mathrm{Br} \mathrm{J}$ Gen Pract. 1994;44:297-300.

51. McHorney CA, Kosinski M, Ware Jr JE. Comparisons of the costs and quality of norms for the SF-36 health survey collected by mail versus telephone interview: results from a national survey. Med Care. 1994;32:551-67.

52. Sumpter RE, McMillan TM. Errors in self-report of post-traumatic stress disorder after severe traumatic brain injury. Brain Inj. 2006;20:93-9.

53. Koponen $\mathrm{S}$, Taiminen $\mathrm{T}$, Portin $\mathrm{R}$, Himanen $\mathrm{L}$, Isoniemi $\mathrm{H}$, Heinonen $\mathrm{H}$, Hinkka S, Tenovuo O. Axis I and II psychiatric disorders after traumatic brain injury: a 30-year follow-up study. Am J Psychiatry. 2002;159:1315-21.

54. Carroll LJ, Cassidy JD, Cancelliere C, Côté P, Hincapié CA, Kristman VL, Holm LW, Borg J, Nygren-De Boussard C, Hartvigsen J. Systematic review of the prognosis after mild traumatic brain injury in adults: Cognitive, psychiatric, and mortality outcomes: Results of the international collaboration on mild traumatic brain injury prognosis. Arch Phys Med Rehabil. 2014;95:S152-73.

55. McHorney CA, Tarlov AR. Individual-patient monitoring in clinical practice: are available health status surveys adequate? Qual Life Res. 1995:4:293-307.

56. Terwee CB, Bot SD, de Boer MR, van der Windt DA, Knol DL, Dekker J, Bouter LM, de Vet HC. Quality criteria were proposed for measurement properties of health status questionnaires. J Clin Epidemiol. 2007;60:34-42.

57. Li CH. Confirmatory factor analysis with ordinal data: Comparing robust maximum likelihood and diagonally weighted least squares. Behav Res Methods. 2016;48:936-49.
58. von Steinbuechel N, Petersen C, Bullinger M. Assessment of health-related quality of life in persons after traumatic brain injury-development of the Qolibri, a specific measure. Acta Neurochir Suppl. 2005;93:43-9.

59. Polinder S, Haagsma JA, van Klaveren D, Steyerberg EW, van Beeck EF. Health-related quality of life after TBI: a systematic review of study design, instruments, measurement properties, and outcome. Popul Health Metr. 2015;13:4.

\section{Submit your next manuscript to BioMed Central and we will help you at every step:}

- We accept pre-submission inquiries

- Our selector tool helps you to find the most relevant journal

- We provide round the clock customer support

- Convenient online submission

- Thorough peer review

- Inclusion in PubMed and all major indexing services

- Maximum visibility for your research

Submit your manuscript at www.biomedcentral.com/submit

) Biomed Central 